\title{
Frequency dependent selection of control sensors in multi-channel acoustic control
}

\author{
Mariano Alvarez Blanco • Edgar Matas • \\ Hadrien Bériot • Bart Peeters • Wim \\ Desmet
}

Received: date / Accepted: date

\begin{abstract}
This paper addresses the problem of reproducing a random pressure field with an array of loudspeakers through direct field acoustic tests. Such field should be representative of the fairing interior acoustic levels generated by the dynamic loading during the launch of a spacecraft. The replication of the acoustic field with loudspeakers is highly dependent on the control strategy used to design the test setup and the signals feeding the acoustic sources. In this paper functional analysis and spectral decomposition theories are used to study the benefit of applying pre-test analysis to set the most optimal control parameters. This paper builds on previous works and assesses the performance of a methodology for frequency dependent distribution of control sensors, which are selected automatically through numerical pre-test analysis. The methodology is evaluated through a numerical model of a direct field acoustic test setup.
\end{abstract}

Keywords Environmental testing · acoustic simulation · direct field · MIMO control $\cdot$ digital twin

Mariano Alvarez Blanco

Siemens Digital Industries Software, Interleuvenlaan 68, 3001 Leuven, Belgium

KU Leuven, Department of Mechanical Engineering, Celestijnenlaan 300 B, B-3001, Heverlee, Belgium

E-mail: mariano.alvarez_blanco@siemens.com

Edgar Matas

Siemens Digital Industries Software

Hadrien Bériot

Siemens Digital Industries Software

Bart Peeters

Siemens Digital Industries Software

Wim Desmet

KU Leuven, Department of Mechanical Engineering

Member of DMMS core lab Flanders Make 


\section{Introduction}

This work explores a model-driven approach to develop control strategies for environmental acoustic tests. This is done in the frame of ground based dynamic environmental testing, which comprises several physical tests for qualification of hardware in the space industry. Such tests are needed to ensure the survivability of satellites during launch, when the payload is exposed to potentially damaging dynamic loads. Acoustic testing is one of the most crucial environmental tests, since it concerns the dominant dynamic load occurring during ignition, lift-off and transonic flight launch stages.

The objective of an environmental acoustic test is to physically reproduce the acoustic field generated during launch inside the launcher fairing, where the satellite is encapsulated. The philosophy behind space hardware design and verification follows the historical assumption that such acoustic field is diffuse. A diffuse acoustic field is theoretically defined as being due to an infinite number of uncorrelated plane waves which, on average, provide equal incident energy from all directions [1]. Since the (theoretically-distant) sources generating these waves are uncorrelated, there would be no interference pattern phenomena in such a sound field and, consequently, the field would be completely homogeneous and isotropic. Ideally, the sound pressure level would be the same at all positions, and temporal correlation functions between linear quantities measured at two points would depend only on the distance between the two points [2].

An approximately diffuse acoustic field may be generated from several loudspeakers positioned in a large anechoic room, as described in an experimental investigation [3]. The anechoic facility provides free-field conditions, which ensure that the acoustic waves are directly arriving from the acoustic sources without boundary reflections. In the practical realization of such a sound field, an array of acoustic sources in an ordinary room can be used to reproduce some of the properties of a diffuse field. There is not direct and unique metric to determine how much diffuseness exists in a given room nor to state how much diffuseness is required for a given application [1]. In previous works, the sound field diffuseness has been evaluated through two kinds of measurements. First, metrics concerning the spatial uniformity of sound pressure level [4]. Secondly, the comparison of measured cross-correlation against a reference, which describes a theoretical diffuse field [5][6][7]. Given the application proposed in this paper, namely the qualification of space hardware, the focus is on the spatial uniformity of sound pressure level, since the most widely required test specification concerns a sound pressure level profile only. Multiple-Input Multiple-Output (MIMO) control is the standard practice in the industry to generate and control the signals driving the loudspeakers surrounding the Device Under Test (DUT). Figure 1 shows an example of an electro-acoustic setup used for the qualification of an antenna reflector subsystem. Although this environmental testing method has been proven successful in reproducing the intended sound field with certain tolerance, previous works have shown 


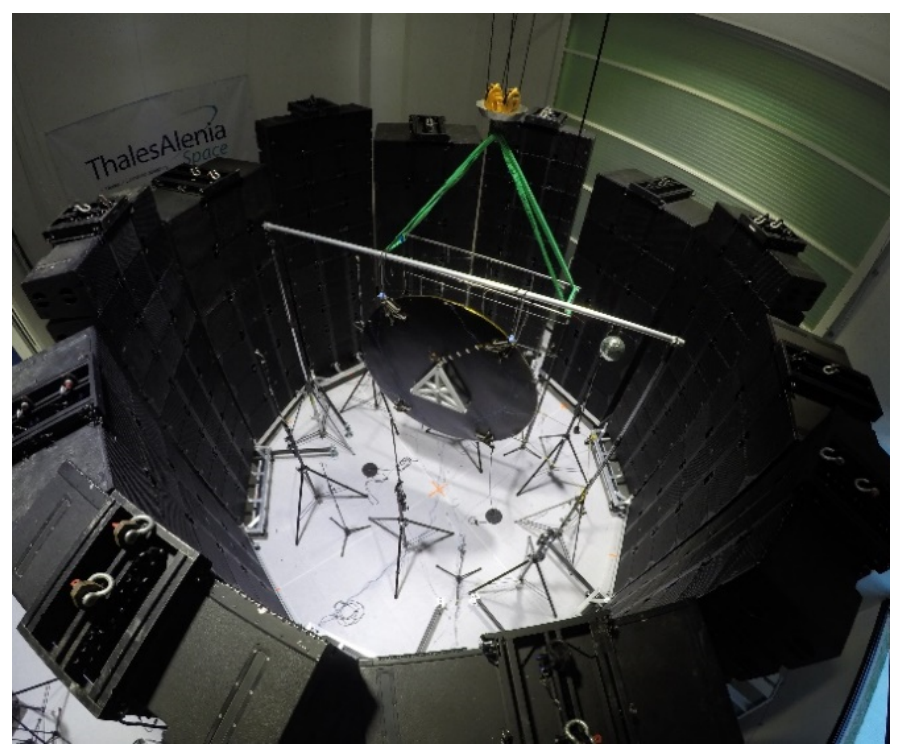

Fig. 1: Direct field Environmental Acoustic Test campaign. Courtesy of Thales Alenia Space (France).

that the efficiency of the test sequence is strongly dependent on the MIMO control strategy $[8][9]$.

Control strategy is a wide concept comprehending several aspects of the test setup, namely: control algorithm principle, electro-acoustic system overdetermination, system identification strategy, test references definition, acoustic sources and control sensors placement, test specimen placement and orientation, drive signals processing and randomization, among others. Given the number of parameters for the control strategy, and the lack of a widely applied recommended practice, some concerns may be raised about potential risks, repeatability and traceability of the test campaigns with direct field excitation. In order to increase the reliability, recent research efforts have introduced numerical simulations to synthesize electro-acoustic frequency response functions [10][7]. This work continues such research line, exploring the application of novel algorithms to further the understanding of the acoustic phenomena producing the structural response of the DUT.

The objective of this research is to develop a Digital Twin to further optimize the design of MIMO control strategies through simulation-based pre-test analysis. The application of a model-driven approach for pre-test analysis intends to improve the efficiency of the setup process, tackling several of the above enumerated control strategy challenges. 


\section{Introduction to Functional Analysis}

The main assumption in this section is that a given acoustic field can be replicated in the interior of a bounded region by a continuous distribution of (e.g. monopole-like) acoustic sources arranged on its boundary. Let $V$ and $\Lambda$ be two simply connected subsets of the three-dimensional space, such that $V \subseteq \Lambda \subset \mathbb{R}^{3}$, and that their boundaries $\partial V$ and $\partial \Lambda$ are smooth (of class $\left.C^{2}[11]\right) . V$ and $\Lambda$ are referred to as the control region and the reproduction region, respectively, while $\partial V$ is referred to as the control boundary. A given choice of $V$ and $\Lambda$ defines a geometrical layout, where the vector $\boldsymbol{z}$ identifies any location in $\mathbb{R}^{3}$, while the vectors $\boldsymbol{l}$ and $\boldsymbol{m}$ identify locations in $\partial V$ and $\partial \Lambda$, respectively. An analytical model of the acoustic field may be used to represent the transfer path, $\boldsymbol{G}(\boldsymbol{z}, \boldsymbol{m}, k)$, where $k=\omega / c$ is the wave number, $\omega=2 \pi f$ the angular frequency, $f$ the frequency and $c$ the speed of sound. Note that the frequency dependence of $\boldsymbol{G}$, and the other variables are suppressed below for notational convenience. For a continuous distribution of sources on $\partial \Lambda$ the source strength can be substituted by the source strength density $u(\boldsymbol{m})$, the strength per unit of area. The pressure field $y(\boldsymbol{z})$ is then assumed to be due to the linear superposition of the fields generated by the layer of sources, and the eventual reflections, at the boundaries of the room and test article. The continuous pressure field can then be described by the integral,

$$
y(\boldsymbol{z})=\int_{\partial \Lambda} G(\boldsymbol{z}, \boldsymbol{m}) u(\boldsymbol{m}) d S(\boldsymbol{m}),
$$

where $d S(\boldsymbol{m})$ represents the infinitesimal fraction of the boundary $\partial \Lambda$. The expression in Eq.(1) is called the acoustic single layer potential [12].

In this context, the acoustic field reproduction problem consists in determining the density $u(\boldsymbol{m})$, such that the reproduced acoustic field $y(\boldsymbol{z})$ is the best approximation of a desired acoustic field $d(\boldsymbol{z})$ in $V$. The operator $S$ has been defined in [11] as the restriction of the single layer potential in Eq.(1) to the boundary $\partial V$, such that,

$$
(S u)(\boldsymbol{l}):=\int_{\partial \Lambda} G(\boldsymbol{l}, \boldsymbol{m}) u(\boldsymbol{m}) d S(\boldsymbol{m}),
$$

Under certain conditions discussed in [11], the desired acoustic field $d(\boldsymbol{z})$ in the interior of $V$ is uniquely determined from a knowledge of its value on the boundary of the control region $\partial V$. This enables to formulate the acoustic field reproduction problem using the operator $S$. The optimal reconstruction of the desired acoustic field is then given by a potential $(\mathrm{Su})$, whose density $u(\boldsymbol{m})$ is the solution of an inverse problem.

\subsection{Spectral decomposition of the operator $\mathrm{S}$}

The solution of the inverse problem can be approached by a spectral decomposition. This consists in representing $S$ as a singular system $\sigma_{n}, q_{n}(\boldsymbol{m}), r_{n}(\boldsymbol{l})$ 
where $\sigma_{n}$ are the singular values of $S$ and $r_{n}(\boldsymbol{l})$ and $q_{n}(\boldsymbol{m})$ are its left and right singular functions, respectively. One of the most powerful features of the spectral decomposition is that any function $u(\boldsymbol{m})$ and any function $y(\boldsymbol{l})$ can be expressed by

$$
\begin{aligned}
u(\boldsymbol{m}) & =\sum_{n=1}^{\infty} q_{n}(\boldsymbol{m})\left\langle q_{n} \mid u\right\rangle_{\partial \Lambda}+\left(P_{N(S)} u\right)(\boldsymbol{m}), \\
y(\boldsymbol{l}) & =\sum_{n=1}^{\infty} r_{n}(\boldsymbol{l})\left\langle r_{n} \mid y\right\rangle_{\partial V}+\left(P_{N\left(S^{*}\right)} y\right)(\boldsymbol{l}),
\end{aligned}
$$

where $\langle\cdot\rangle_{D}$ is the scalar product on a set $D$, the superscript $\cdot{ }^{*}$ represents, for a scalar the complex conjugate and for an operator its adjoint, and $P_{N(S)}$ and $P_{N\left(S^{*}\right)}$ are the orthogonal projection operators onto the null-space of $S$ and $S^{*}$, respectively.

This implies that in the case of $S$ and $S^{*}$ injective, any square integrable function $y(\boldsymbol{l})$ representing the acoustic field on $\partial V$, can be expressed as a series of the singular functions $r_{n}(\boldsymbol{l})$. Analogously, any square integrable function $u(\boldsymbol{m})$ defining the source strength on $\partial \Lambda$ can be expressed as a series of $q_{n}(\boldsymbol{m})$. This could be interpreted as the modal decomposition of the source strength $u(\boldsymbol{m})$ and of the pressure profile $y(\boldsymbol{l})$ in the spatial domain.

As proposed in [11], the action of the operator $S$ on $u(\boldsymbol{m})$ can be then expressed by

$$
(S u)(\boldsymbol{l})=\sum_{n=1}^{\infty} r_{n}(\boldsymbol{l}) \sigma_{n}\left\langle q_{n} \mid u\right\rangle_{\partial \Lambda} .
$$

Eq.(5) suggests that the reproduction of the acoustic field could be realized by synthesizing the desired acoustic field after summing the infinite, but countable, set of scaled orthogonal functions for the continuous problem.

2.2 Solution of the theoretical inverse problem

If an exact solution to the integral Eq.(5) exists, this is given by

$$
u(\boldsymbol{m})=\sum_{n=1}^{\infty} q_{n}(\boldsymbol{m}) \frac{1}{\sigma_{n}}\left\langle r_{n} \mid d\right\rangle_{\partial \Lambda},
$$

which provides an expression of the source strength function $u(\boldsymbol{m})$ in terms of singular functions and singular values of $S$ [11]. In this representation it is important to remark that such singular functions and singular values do not depend on the desired acoustic field $d(\boldsymbol{z})$. However, the existence of an exact solution depends on the properties of the desired acoustic field. 
2.3 Discretization of the acoustic field reproduction problem

The spectral decomposition introduces the notion of singular values and left/rightsingular vectors of the compact operator $S$ on a Hilbert space, and shows that they have a discrete spectrum (i.e. countable singular values). In a most practical scenario, for a discrete problem where $\mathrm{S}$ takes the form of a complex matrix, $\boldsymbol{G}$, the Singular Value Decomposition (SVD) is used for a similar factorization. In this context, the SVD can be interpreted as the generalization of the spectral decomposition, the latter for positive semi-definite normal matrices (symmetric matrices with positive eigenvalues). However, the use of a finite number of acoustic sources instead of a continuous distribution can generate reproduction artifacts [11]. These artifacts are mostly related to the problem of spatial aliasing. This and other phenomena are well-known and discussed also in the literature dedicated to sampling theory [11][13][14].

\section{Simulation}

A model of an electro-acoustic plant can be used to discretize the problem. This model simulates a test setup comprising 6 stacks of 10 loudspeakers, and 6 stacks of 6 sub-woofers, as shown in figure 2. The 12 stacks are alternated with a circular distribution of $5.28 \mathrm{~m}$ radius. The sources are surrounding a validated model of the DUT, a telecom reflector shell of an antenna subsystem. This model has been simulated under free-free conditions. The center of the DUT is placed $2.1 \mathrm{~m}$ above the origin of the coordinate system. The local normal at the center of the antenna is parallel to the XY planes and rotated 30 degrees around the $\mathrm{Z}$ axis in positive direction from the $\mathrm{X}$ axis. This way, positive $\mathrm{Z}$ direction defines the vertical direction and the XY plane define the horizontal plane. A set of 3392 microphone elements are distributed along two perpendicular planes and at discrete points in the field, as shown in figure 3 . Each plane has a total 1681 microphone points distributed in a quadrangular grid of $0.05 \mathrm{~m}$. The total size of each plane is $2 \mathrm{~m} \times 2 \mathrm{~m}$. The first plane is horizontal and passes through the center of the antenna, while the second plane is a vertical plane placed $0.24 \mathrm{~m}$ in front of the antenna. In addition, 30 microphones are distributed around the antenna as shown in green also in figure 3. The acoustic mesh and the vibro-acoustic model were set up in the commercial Finite Element software Simcenter3D v12.0.1. The simulation was then solved in NX Nastran solver v12.0.1 for frequencies between $25 \mathrm{~Hz}$ and $800 \mathrm{~Hz}$ with a frequency step of $3.125 \mathrm{~Hz}$, resulting in a total of 249 frequency lines.

All the vibro-acoustic simulations are using adaptive Finite Elements (FEMAO) for the acoustic elements. This technology uses higher order shape functions (from order 1 to 10) that automatically adapt to the frequency that is being solved in order to properly capture the acoustic waves at each element. This results in an optimized computational time for the whole frequency range while allowing to increase the element size in the model [15]. A proprietary 

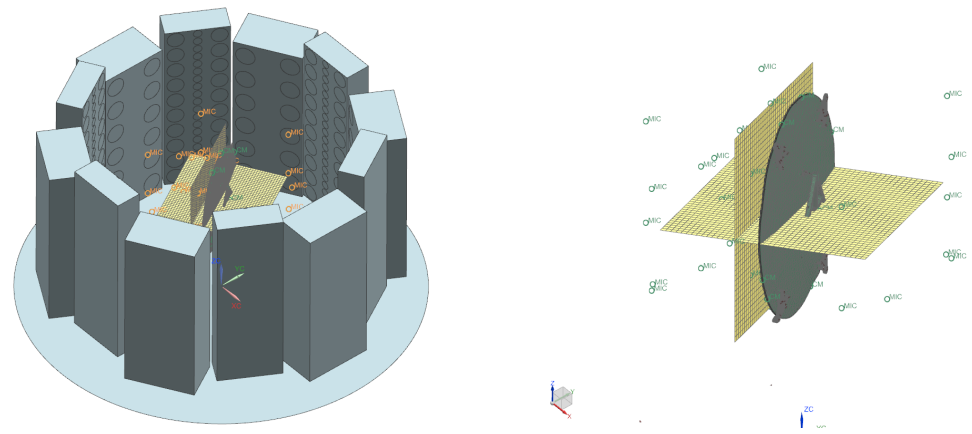

Fig. 2: CAD of electro-acoustic system Fig. 3: Structural Nastran model of for Direct Field Environmental Acous- telecom reflector shell of an antenna tic Testing [7]. subsystem [7]. Courtesy of Thales Alenia Space (France).

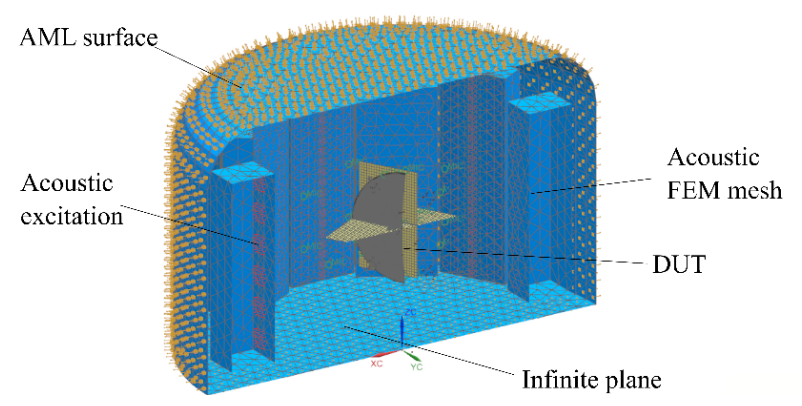

Fig. 4: Vibro-acoustic model with plane cut [7].

automated version of the Perfectly Matched Layer, referred to as the AML (Automatically Matched Layer) is defined at the boundary of the FEM domain, in order to avoid reflections and to ensure the propagation of waves in a relatively small FEM domain. The floor as well as all the surfaces of the speakers are considered perfectly rigid. Figure 4 shows a cut of the acoustic FEM mesh, which in total has 87223 tetrahedral elements.

\section{Active Acoustic Control}

In the following a brief introduction to active acoustic control is provided. The objective is to extend the introduction of functional analysis in an active random control context. Such extension of the theoretical background helps to understand how certain selection of control microphones may affect the control performance during a qualification test. 


\subsection{Optimal Control}

In the practical reproduction of the acoustic field, active control at $L$ control microphones is intended by generating a set of $M$ white noise signals, which are then feeding a matrix of control filters to drive the loudspeakers. The frequency domain control approach described below is based on the work of Elliot et al. [6] and aims at the synthesis of a random pressure field with prescribed spatial correlation properties, where the targeted pressure excitation is modelled as random stationary processes and represented by the spectral density matrix,

$$
\boldsymbol{S}_{d d}=E\left[\boldsymbol{d d}^{H}\right]
$$

where $\boldsymbol{S}_{d d} \in \mathbb{C}^{L x L}$ and $\boldsymbol{d} \in \mathbb{C}^{L x 1}$ is the vector of Fourier transforms, at a single frequency, of the desired outputs from the microphones array, E denotes the expectation operator and the superscript ${ }^{H}$ represents the complex-conjugate transpose operator. In this description of the stochastic controller, the theoretical aspects of adaptive algorithms are not discussed. The reader is referred to [16][17][18] for additional details on the techniques to adapt the matrix of control filter along the test.

The vector $\boldsymbol{y} \in \mathbb{C}^{L x 1}$ of output spectra from the microphones array can be written as

$$
\boldsymbol{y}=\boldsymbol{G W} \boldsymbol{x},
$$

where $\boldsymbol{G} \in \mathbb{C}^{L x M}$ might be the plant modelled in section 3 and $\boldsymbol{x} \in \mathbb{C}^{M x 1}$ is the vector of white noise signal spectra used to generate the inputs to the loudspeakers, $\boldsymbol{u} \in \mathbb{C}^{M x 1}$, via the matrix of filter frequency responses denoted $\boldsymbol{W} \in \mathbb{C}^{M x M}$ as

$$
\boldsymbol{u}=\boldsymbol{W} \boldsymbol{x} .
$$

The vector of error signals, $\boldsymbol{e} \in \mathbb{C}^{L x 1}$, defined as the difference between the desired output signals, $\boldsymbol{d}$, and the measured output signals, $\boldsymbol{y}$, can be written as

$$
e=\boldsymbol{d}-\boldsymbol{y} \text {. }
$$

If these error signals can be made small, the acoustic pressure generated by the loudspeakers at the control microphones must be statistically equivalent to those of the desired pressure field, such that,

$$
\boldsymbol{S}_{y y}=E\left[\boldsymbol{y} \boldsymbol{y}^{H}\right]=\boldsymbol{S}_{d d}
$$

The plant modelled in section 3 might be also represented using the SVD as

$$
\boldsymbol{G}=\boldsymbol{R} \boldsymbol{\Sigma} \boldsymbol{Q}^{H}
$$

where $\boldsymbol{R} \in \mathbb{C}^{L x L}$ is the unitary matrix of complex left singular vectors of $\boldsymbol{G}$, $\boldsymbol{Q} \in \mathbb{C}^{M x M}$ is the unitary matrix of complex right singular vectors of $\boldsymbol{G}$, and 
$\boldsymbol{\Sigma} \in \mathbb{C}^{L x M}$ is the matrix containing the singular values, $\sigma_{i}$ for $i=1 \ldots M$, of $\boldsymbol{G}$. Substituting Eq.(8) to (9) and (12) into the general expression of the error in Eq.(10) and pre-multiplying by $\boldsymbol{R}^{H}$ yields

$$
\boldsymbol{R}^{H} \boldsymbol{e}=\boldsymbol{R}^{H} \boldsymbol{d}-\boldsymbol{\Sigma} \boldsymbol{Q}^{H} \boldsymbol{W} \boldsymbol{x}
$$

where the property $\boldsymbol{R}^{H} \boldsymbol{R}=\boldsymbol{I}$ was used, and $\boldsymbol{I}$ is the identity matrix. Then, the transformed set of signals

$$
\begin{aligned}
& \boldsymbol{s}=\boldsymbol{R}^{H} \boldsymbol{e}, \\
& \boldsymbol{p}=\boldsymbol{R}^{H} \boldsymbol{d}, \\
& \boldsymbol{v}=\boldsymbol{Q}^{H} \boldsymbol{u},
\end{aligned}
$$

enable to write Eq.(10) as

$$
s=p-\Sigma v .
$$

The diagonal nature of the $\boldsymbol{\Sigma}$ matrix means that each of the transformed error signals is either a function of only a single transformed drive signal from the control filter [18], so that

$$
s_{l}=p_{l}-\sigma_{l} v_{l}, \quad \text { for } \quad 1 \leq l \leq M,
$$

or is completely unaffected by the drive signals, so that

$$
s_{l}=p_{l}, \quad \text { for } \quad M+1 \leq l \leq L,
$$

where the $l$-th singular values quantifies how well the $l$-th transformed drive signals couple into the $l$-th transformed error signal.

Eq.(17) to (19) shows that the SVD enables to transform the physical error signals and split the components of the desired signals that are in the rankspace of $\boldsymbol{G}$, and can be controlled, from those that are in the null-space of $\boldsymbol{G}$ and thus cannot be controlled.

\subsection{Modal decomposition}

$\boldsymbol{R}$ and $\boldsymbol{Q}$ in Eq.(12) represent rotations or reflections in a Cartesian space and their columns, the left and right singular vectors $\boldsymbol{r}_{i} \in \mathbb{C}^{L x 1}$ and $\boldsymbol{q}_{i} \in \mathbb{C}^{L x 1}$, respectively, are orthogonal to each other. These singular functions obtained after applying the SVD to $\boldsymbol{G}$ have been interpreted to as acoustic pressure modes and source modes (or loudspeakers modes) [19][11]. For example, $\boldsymbol{q}_{1}$, the first loudspeakers mode, is often constituted by identical elements, thus corresponding to all loudspeakers acting in phase and with the same amplitude. Following this analysis, the operation $\boldsymbol{Q}^{H} \boldsymbol{u}$ can be interpreted as a modal 
decomposition of the vector $\boldsymbol{u}$. The coefficients obtained from the product (or orthogonal projection) $\boldsymbol{q}_{i} \boldsymbol{u}$ shows how much of the array mode $\boldsymbol{q}_{i}$ is present in $\boldsymbol{u}$ [11]. The power of the SVD then originates from the fact that each mode $\boldsymbol{q}_{i}$ is transformed by $\boldsymbol{G}$ into the mode $\boldsymbol{r}_{i}$. This, together with the mutual orthogonality of the modes, implies that the mode $\boldsymbol{r}_{i}$ depends on the mode $\boldsymbol{q}_{i}$ and on this mode only, holding that

$$
\boldsymbol{G} \boldsymbol{q}_{i}=\sigma_{i} \boldsymbol{r}_{i}
$$

However, spatial aliasing may compromise this one-to-one transformation due to modal leakage if the sampling schemes from the continuous to the discrete domain are not optimal. For example, in [11] was shown how the sampling of $u(\boldsymbol{m})$ into $\boldsymbol{u}$ causes aliasing artifacts, which may potentially degrade the accuracy of the acoustic field reproduction. Analogous considerations can be done for the sampling of $y(\boldsymbol{l})$ into $\boldsymbol{y}$. Furthermore, the concept of modal assurance criterion $(\boldsymbol{M})$ [20], or orthogonality matrix as similarly defined in [11], can be borrowed to study the behaviour of the pressure modes in the selection of control microphones. This is because if $T$ microphones in the field are available and $L<T$ need to be selected for control, this can be interpreted as a sampling task. Then, the modal assurance criterion can serve as a tool to compare the quality of different sampling schemes. The modal assurance criterion between two pressure sampled modes $\boldsymbol{r}_{i} \in \mathbb{C}^{L x 1}$ and $\boldsymbol{r}_{j} \in \mathbb{C}^{L x 1}$ can be written as

$$
M_{i j}=\frac{\left|r_{i} r_{j}^{H}\right|^{2}}{\left(r_{i}^{H} r_{j}\right)\left(r_{i} r_{j}^{H}\right)},
$$

where $|\cdot|$ corresponds to the 2 -norm vector norm. If $\boldsymbol{M}$ is an identity matrix, then the orthogonality of the modes is preserved. On the contrary, the presence of non-zero off-diagonal terms indicates lack of orthogonality between re-sampled modes. In simple terms, the modes can no longer be controlled independently of one another. This phenomenon has been referred to as modal leakage and relates to the phenomenon known as spatial aliasing [11].

Figure 5 shows the effect produced by $\boldsymbol{q}_{1}$ at $\boldsymbol{G}$, for example, for the frequencies $31.5 \mathrm{~Hz}, 63 \mathrm{~Hz}, 125 \mathrm{~Hz}$ and $250 \mathrm{~Hz}$. Furthermore, figure 6 shows the effect produced by higher order modes at frequency $31.5 \mathrm{~Hz}$, where the discontinuities in the colormaps are due to reflection and radiation provided by the antenna reflector. Note that besides the mode number, figure 6 shows the relative efficiency of each mode, given by $\sigma_{i} / \sigma_{1}$. The singular values $\sigma_{i}$, real and positive scalars in Eq.(20), represent the amount of amplification or attenuation that each mode $\boldsymbol{q}_{i}$ (of unitary norm) undergoes for the transformation $\boldsymbol{G}$. If the singular value $\sigma_{i}$ is large, then the mode $\boldsymbol{q}_{i}$ is efficient, in energetic terms, and it is not efficient if $\sigma_{i}$ is small.

The modal decomposition of the acoustic field can be used to study the capability of $\boldsymbol{G}$ to reproduce a desired field, $\boldsymbol{d}$, through the combination of the available modes. The availability and efficiency of such modes depends on 

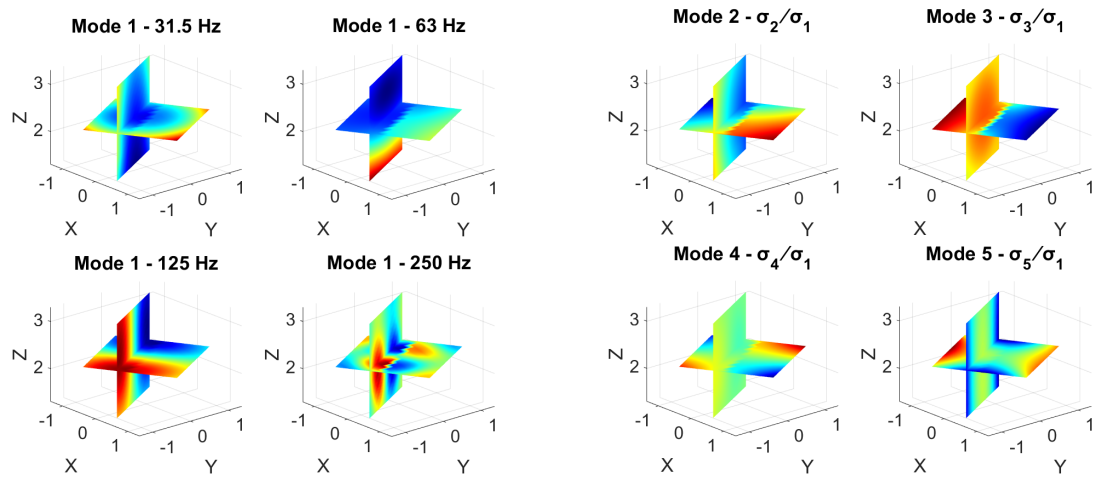

Fig. 5: First mode for frequencies 31.25 Fig. 6: Higher order modes at fre$\mathrm{Hz}, 62.5 \mathrm{~Hz}, 125 \mathrm{~Hz}$ and $250 \mathrm{~Hz}$. quency $31.5 \mathrm{~Hz}$.

the rank-space spanned by the columns of $\boldsymbol{G}$, which in turn, depends on the location of the microphones and loudspeakers.

\section{Optimisation algorithms}

The optimisation algorithms described below are presented as part of a pre-test analysis, which intends to enhance a MIMO control strategy. The algorithms enable automatic selection of control microphones. For this purpose, the assumption is made that $T$ amount of microphones are available, comprising $L$ control microphones plus an additional set of $(T-L)$ monitoring microphones. By selecting optimal control microphones, it is expected to enhance the capability of the electro-acoustic system to reproduce a desired pressure field, the test specification, through an efficient combination of loudspeakers modes, as described in the previous section.

\subsection{Full frequency bandwidth optimisation}

This approach intends to find one optimal spatial distribution of microphones to control the electro-acoustic plant in the full frequency bandwidth, while the position of the loudspeakers is fixed. This can be achieved by trying different sets of control microphones, which translates into iteratively checking the column-space of the control plant to screen out under-performing control layouts. To avoid manual selection of control sensors, an exhaustive search could be used. It would consist in systematically enumerating all possible candidates for the solution of the problem, and checking whether each candidate satisfies the problem's statement [18]. In this approach a candidate is defined as a combination without repetition of $L$ control microphones out of the $T$ microphones placed in the test volume, whereas the problem's statement concerns 
the reproduction of a sound field with uniform spatial distribution of sound pressure level. This is then a deterministic problem with a set of finite discrete solutions, including a global optimum, namely, the optimal set of control sensors. However, for certain $T$ and $L$, the number of combinations without repetition quickly raises, making an exhaustive search infeasible due to simulation time constraints. Therefore, in this work a reduced number of 4,000 candidates randomly selected from the discrete search space are used for the optimisation.

This optimisation process consists of the five steps explained below and shown in the scheme of figure 7 .

1. Definition of the inputs $\boldsymbol{G}^{\text {init }} \in \mathbb{C}^{T x M}$ and $\boldsymbol{S}_{d d}^{\text {init }} \in \mathbb{C}^{T x T}$, where the superscript .init refers to the use of the $T$ microphones. $\boldsymbol{S}_{d d}^{\text {init }}$ is the matrix of initial test references, the outcome of the Unconstrained Projection Algorithm (UPA), as described in [21].

2. Definition of candidates. Given a number of control microphones $L$, where $L<T$, each candidate consists of $\boldsymbol{G}_{i}^{\text {init }} \in \mathbb{C}^{L x M}$ and $\boldsymbol{S}_{d d_{i}}^{\text {init }} \in \mathbb{C}^{L x L}$, where the subindex $\cdot_{i}$ refers to the candidate number. $\boldsymbol{G}_{i}^{i n i t}$ and $\boldsymbol{S}_{d d_{i}}^{i n i t}$ are submatrices of $\boldsymbol{G}^{\text {init }}$ and $\boldsymbol{S}_{d d}^{\text {init }}$, respectively.

3. Prediction of the pressure responses at the $T$ microphones as

$$
\boldsymbol{S}_{y y_{i}}^{\text {pred }}=\boldsymbol{G}^{\text {init }}\left(\boldsymbol{G}_{i}^{i n i t}\right)^{\dagger} \boldsymbol{S}_{d d_{i}}^{\text {init }}\left[\boldsymbol{G}^{\text {init }}\left(\boldsymbol{G}_{i}^{i n i t}\right)^{\dagger}\right]^{H}
$$

where the superscript ${ }^{\dagger}$ corresponds to the pseudo-inverse operator. The computation in Eq.(22) is performed for each frequency of the bandwidth. Then, the predicted responses are represented as sound pressure levels in 1/3rd octave bands and used to compute two descriptors: $E_{\text {bands }}$ and the Root Mean Square Error (RMSE). The first corresponds to the amount of $1 / 3$ rd octave bands exceeding or below a frequency dependent tolerance (e.g. $-1 /+2 \mathrm{~dB}$ with respect to the test specification at $100 \mathrm{~Hz}$ ). And RMSE describes the deviation of the spatial average of sound pressure level from the test specification. During the optimisation procedure, these descriptors are stored and linked to each candidate.

4. The process is repeated iteratively until a fixed number of candidates are evaluated (i.e. $\mathrm{n}_{\max }=4,000$ ).

5. Finally, one candidate leading to the minimum $E_{b a n d s}$ is selected. In case there are several candidates with the same $E_{\text {bands }}$, the one with minimum RMSE between them is chosen. Since RMSE is not sensitive to large sound pressure level dispersion at each band, $E_{\text {bands }}$ takes precedence. This prioritization guarantees that both, sound pressure level responses at each microphone as well as spatial average are as close as possible to the test specification. 

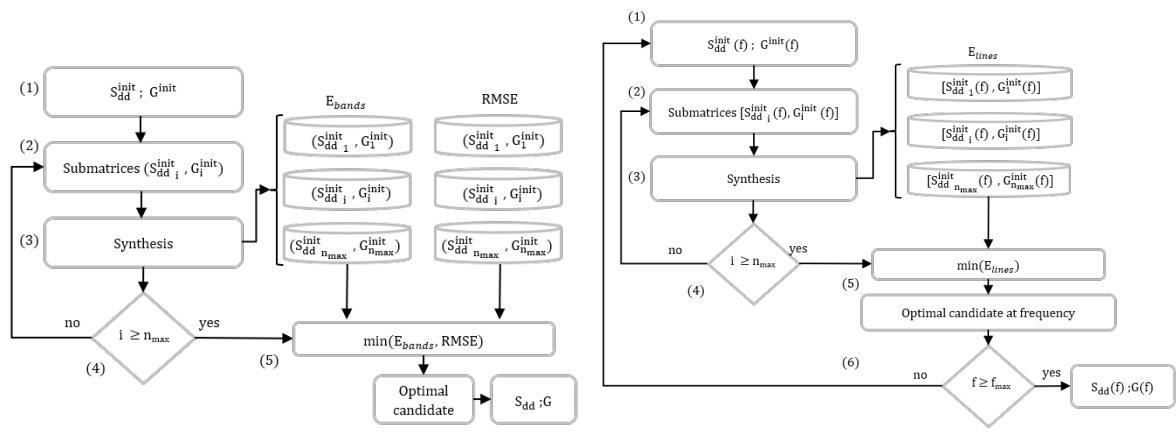

Fig. 7: Block diagram of full band- Fig. 8: Block diagram of frequency dewidth optimisation algorithm. pendent optimisation algorithm.

\subsection{Frequency dependent optimisation}

In a frequency dependent approach the optimisation procedure is similar. However, the objective is to find an optimal candidate for each frequency. Then, starting from $T$ microphones distributed in the field it is intended to find optimal subsets of $L$ control microphones enabling to uniformly reach the sound pressure level responses desired at each frequency. It is expected that such optimal candidates will combine in the most efficient way the so-called loudspeaker modes for each frequency.

As in case of the full frequency bandwidth, this optimisation can also be conducted as an exhaustive search, but considering a randomly selected number of candidates. The process consists of the six steps explained below and shown in the scheme of figure 8 .

1. Similarly to the previous first item, the inputs to the algorithm consist of $\boldsymbol{G}^{\text {init }}$ and $\boldsymbol{S}_{d d}^{\text {init }}$. However, only for the evaluated frequency, $f$.

2. For this comparative analysis between optimisation algorithms, the same 4, 000 candidates are defined, and sub-matrices of $\boldsymbol{G}^{\text {init }}$ and $\boldsymbol{S}_{d d}^{\text {init }}$ are obtained for the evaluated frequency.

3. Prediction of the pressure responses at the $T$ microphones also follow Eq.(22). However, note that only responses at frequency $f$ are taken into consideration. The descriptor used to quantify the control performance provided by each candidate is $E_{\text {lines }}$, which quantifies the amount of narrowband responses out of tolerance for the frequency line.

4. The process is repeated until the 4,000 candidates are evaluated.

5. The candidate with minimum $E_{\text {lines }}$ is selected as optimal for the evaluated frequency $f$.

6. The process is repeated until each frequency of the bandwidth is evaluated, obtaining an optimal candidate for each of these frequencies. 


\section{Results}

In the following, the results obtained by applying the optimisation algorithms are presented. The model described in Section 3 is used to simulate the behaviour of the electro-acoustic plant when it is configured with $M=6$ inputs, where each input is feeding two stacks, one of loudspeakers and one of subwoofers. The output of the simulation are the pressure responses at $T=98$ out of the 3392 available microphone elements. The control algorithm described in Section 4 needs a control plant of $M=6$ inputs and $L=12$ outputs to design the signals $\boldsymbol{u}$. The number of outputs is limited to 12 in order to reproduce the conditions of a physical test, where a limited number of control microphones is available. The optimisation algorithms described in section 5 are used to conduct:

- Case study 1 , which aims to minimise $E_{\text {bands }}$ to obtain a unique set of $L$ control microphones to control the bandwidth from $25 \mathrm{~Hz}$ to $800 \mathrm{~Hz}$.

- Case study 2, which aims to minimise $E_{\text {lines }}$ to obtain 249 sets of $L$ control microphones, one for each frequency spanning the bandwidth $25 \mathrm{~Hz}$ to 800 $\mathrm{Hz}$, with frequency resolution $3.125 \mathrm{~Hz}$.

\subsection{Case study 1: full-band optimisation}

The first case study is used as reference, since it was applied in a previous work [22]. In this way, it enables to compare with case 2 , and to evaluate if added value is provided by the frequency dependent optimisation.

On the left, figure 9 shows in red the spatial distribution of the optimal control sensors for this case study. In the same figure, the blue dots show the discarded control locations, after 4,000 possible candidates where simulated and evaluated. The 98 measurements points enable the computation of $\boldsymbol{r}_{i}^{\text {init }} \in$ $\mathbb{C}^{T x 1}$, where $i=1, \ldots, 98$. The modal assurance criterion matrix in figure 10 show, for this optimal choice of microphones, the correlation pattern between the first 30 modes at frequencies $31.25 \mathrm{~Hz}, 62.5 \mathrm{~Hz}, 125 \mathrm{~Hz}$ and $250 \mathrm{~Hz}$. Dark blue values in the off-diagonal elements of the matrix represent low correlation, while bright yellow flags high correlation. The patterns depend on the sampling scheme given by the distribution of the control sensors. Since the plant $\boldsymbol{G}^{\text {init }} \in$ $\mathbb{C}^{T x M}$ has rank 6 , a maximum of 6 modes may be ideally uncorrelated in the matrix (modes 1 to 6 ). On the right, figure 9 shows the results for the worst candidate. The microphones in this case are mostly clustered in the vertical plane, hindering the capability to properly control the field. This is shown by the matrix $\boldsymbol{M}$ in figure 11, where a much higher degree of correlation than in figure 10 is presented by the modes, $\boldsymbol{r}_{i}^{\text {init }}$, for all frequencies.

Figures 12 and 13 present the predicted sound pressure levels from step (3) of the optimisation algorithm. The blue curve in each figure corresponds to the test specification, while the green ones present the tolerance levels. The red ones are the abort levels, as would be set for safety in a physical test. Finally, the dashed black curve corresponds to the spatial average of predicted 


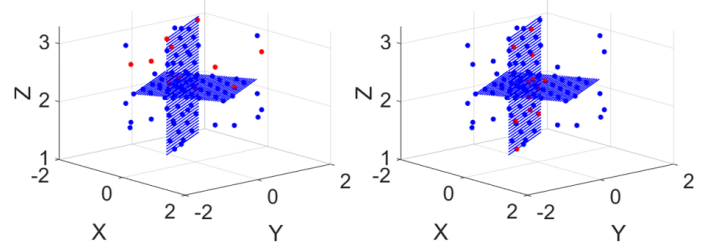

Fig. 9: Spatial distribution of microphones in full-band optimisation. Left: Optimal. Right: worst.

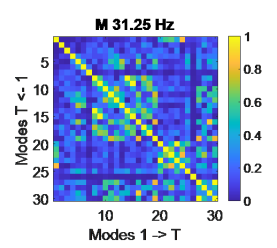

$M 125 \mathrm{~Hz}$

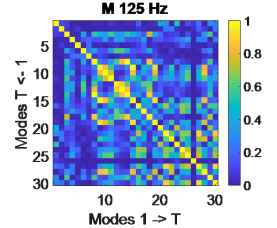

$\stackrel{10}{\operatorname{Modes}} \underset{1 \rightarrow \mathrm{T}}{20}$

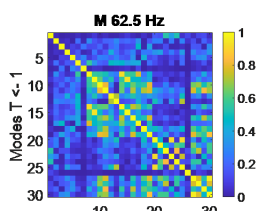

Modes $1 \rightarrow T$
$M 250 ~ H z$

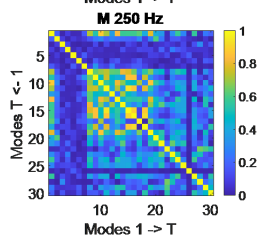

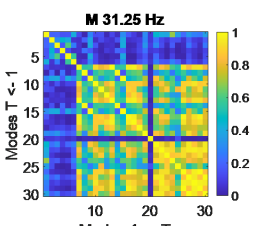

$\stackrel{10}{20} \underset{\text { Modes } 1 \rightarrow T}{1 \rightarrow}$

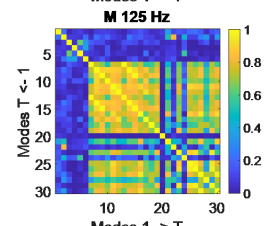

$\stackrel{10}{20} \underset{M o d e s}{1} \rightarrow \mathrm{T}$

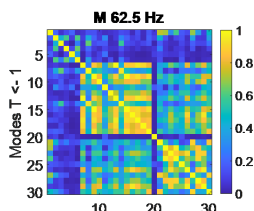

$\stackrel{10}{20} \underset{\text { Modes } 1 \rightarrow T}{ }$

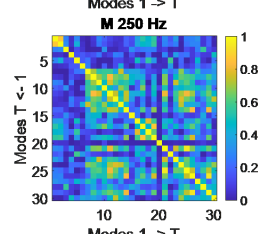

$\underset{\text { Modes }}{10} \stackrel{20}{\rightarrow}$

Fig. 10: Modal assurance criterion for Fig. 11: Modal assurance criterion for optimal candidate in full band optimi- worst candidate in full band optimisasation. tion.

sound pressure level. Figure 12 concerns the optimal candidate. On top, the figure shows the sound pressure level results for the $L$ control microphones. Note that for each $1 / 3$ rd octave band the number of bands out of tolerance, $E_{\text {bands }}^{L}$, is displayed, as well as the standard deviation, $E_{s t d}^{L}$. On the bottom of figure 12 , the results for the $T$ microphones are shown, where $E_{\text {bands }}^{T}$ is the number of bands out of tolerance for each $1 / 3$ rd octave band. These values are used for the quantification of $E_{\text {bands }}$ as cost function in the optimisation process, in this case $E_{\text {bands }}$ rises to 160 bands.

In figure 13 the results produced by the candidate with the largest $E_{\text {bands }}$ are presented. These sound pressure level responses correspond to the worst candidate, which is shown on the right of figure 9 . Note that in the bottom of figure $13 E_{\text {bands }}$ rises to 539 bands. For the bandwidth considered in this work and the value of $T$, this result represents $34 \%$ of the total number of displayed $1 / 3$ rd octave bands. Over- and under-testing levels can be observed of up to $15 \mathrm{~dB}$ with respect to the test specification. And the spatial average of sound pressure level also presents large over-testing. However, note that the responses of the control microphones on the top part of figure 12 and 13 are actually good. This is because the controller designs the drive signals, $\boldsymbol{u}$, 

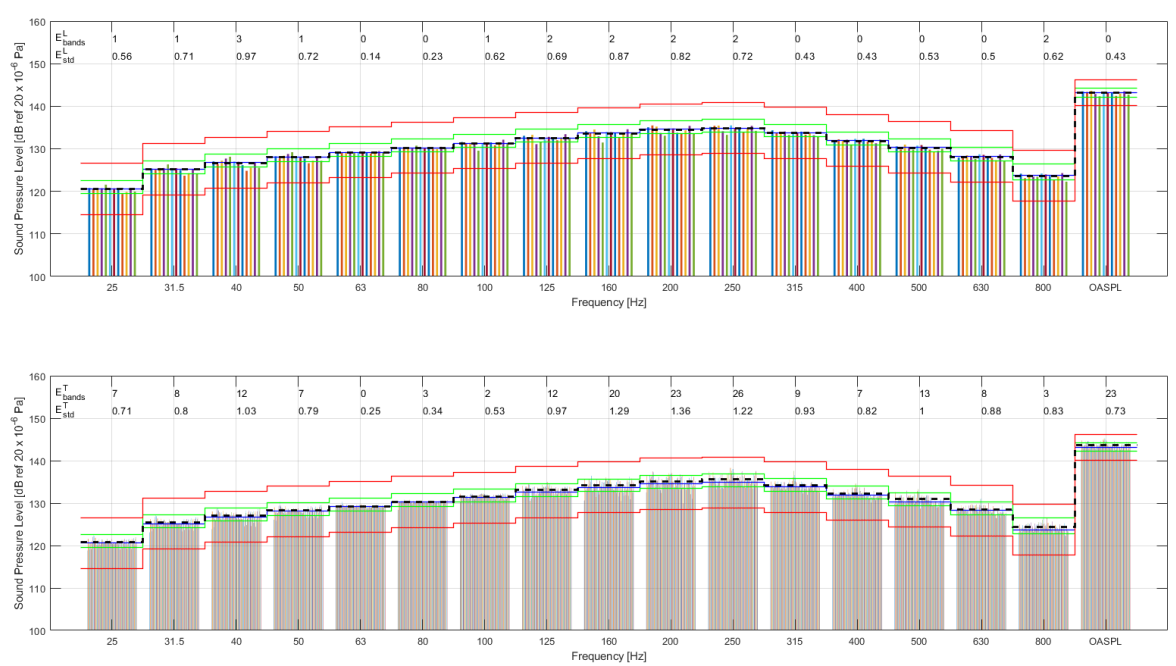

Fig. 12: Sound pressure levels for optimal candidate in full-band optimisation. Top: $L$ control microphones. Bottom: $T$ microphones.
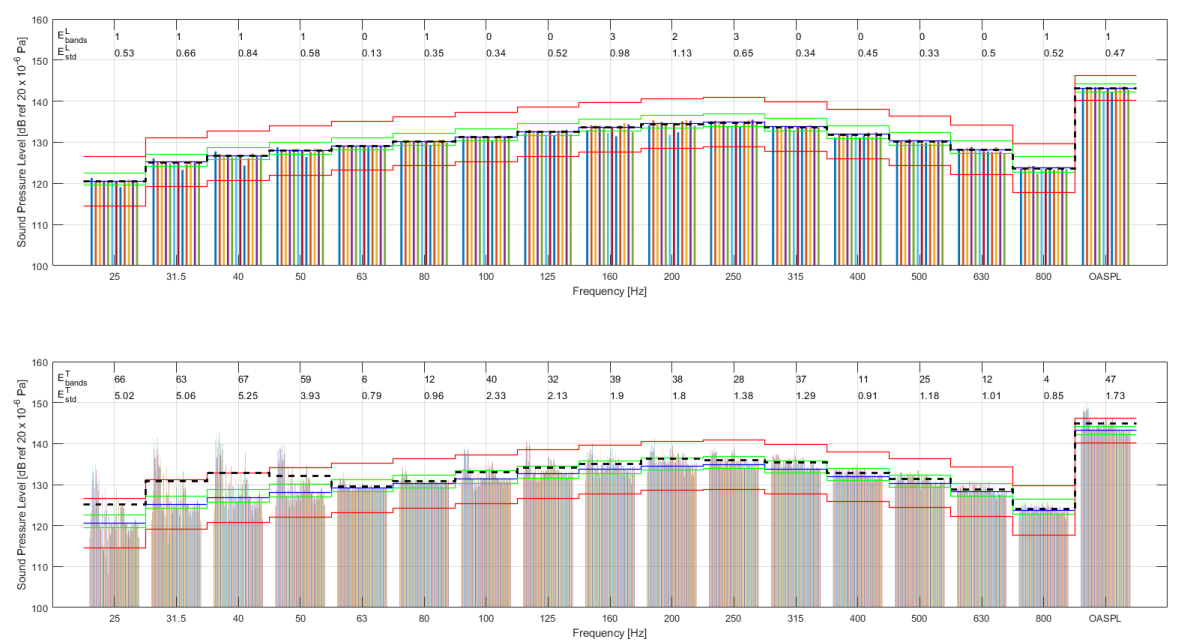

Fig. 13: Sound pressure levels for worst candidate in full-band optimisation. Top: $L$ control microphones. Bottom: $T$ microphones.

with this objective (through the matrix of filters $\boldsymbol{W}$ ), while the enhancement of sound pressure level uniformity in the rest of the measured field $(T-L)$ is given, in figure 12, by the optimal candidate provided by the algorithm. 


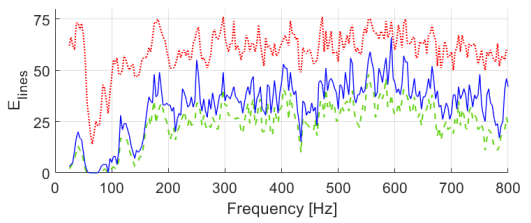

Fig. 14: $E_{\text {lines }}$ for optimisation approaches.

6.2 Case study 2: frequency dependent optimisation

The second case study explores the application of the frequency dependent optimisation. In this approach the objective is to find an optimal set of $L$ microphones for each of the 249 frequencies spanning the control bandwidth.

The cost function in this case is $E_{\text {lines }}$, since a narrow-band descriptor is necessary. In figure $14, E_{\text {lines }}$ quantifies the number of frequency lines of pressure power spectra below or above the test tolerance. This descriptor is displayed as reference in blue for the optimal candidate of the full-band optimisation presented in Section 6.1. The green dashed curve shows that the optimal candidate of the frequency dependent optimisation can reduce $E_{\text {lines }}$ with respect to the reference case. In addition, the results obtained for the worst candidate in the frequency dependent optimisation is shown in red.

Figure 15 shows in red the optimal spatial distribution of control microphones for frequencies $31.25 \mathrm{~Hz}, 62.5 \mathrm{~Hz}, 125 \mathrm{~Hz}$ and $250 \mathrm{~Hz}$. The same distribution of control microphones can be found for $31.25 \mathrm{~Hz}, 62.5 \mathrm{~Hz}$. This may be due to the harmonic relationship between the frequencies. Furthermore, may be due to the relation between the wavelengths, $\lambda=c / f$, and the relatively short distance between microphones. Since the wavelengths for frequencies $31.25 \mathrm{~Hz}$ and $62.5 \mathrm{~Hz}$ are in general larger than the distance between the microphones, the measurements are likely to be similar. Therefore, explaining the same outcome of the optimisation for both frequencies.

Figure 16 presents the modal assurance criterion matrices for the optimal candidate at frequencies $31.25 \mathrm{~Hz}, 62.5 \mathrm{~Hz}, 125 \mathrm{~Hz}$ and $250 \mathrm{~Hz}$. The correlation patterns are in this case produced by different sampling schemes for each frequency, however, leading to similar matrices to the ones in figure 10. This might be expected at frequencies $31.25 \mathrm{~Hz}, 62.5 \mathrm{~Hz}$, because the optimal spatial distribution of control microphones is the same than on the left of figure 9 , another proof of the low sensitivity of the optimisation methodology to frequencies with large wavelength. Although with a similar pattern, at $250 \mathrm{~Hz}$ the degree of correlation between modes in figure 16 seems to be higher than in figure 10 .

Figure 17 shows in red the spatial distribution of control microphones for the worst candidate. A general observation, similar to figure 9 on the right, is that the control microphones are mostly clustered in the vertical plane. This vertical distribution presents poor control performance because each of the $M=6$ drive signals feeding the sources are assigned to full (also vertical) stacks 

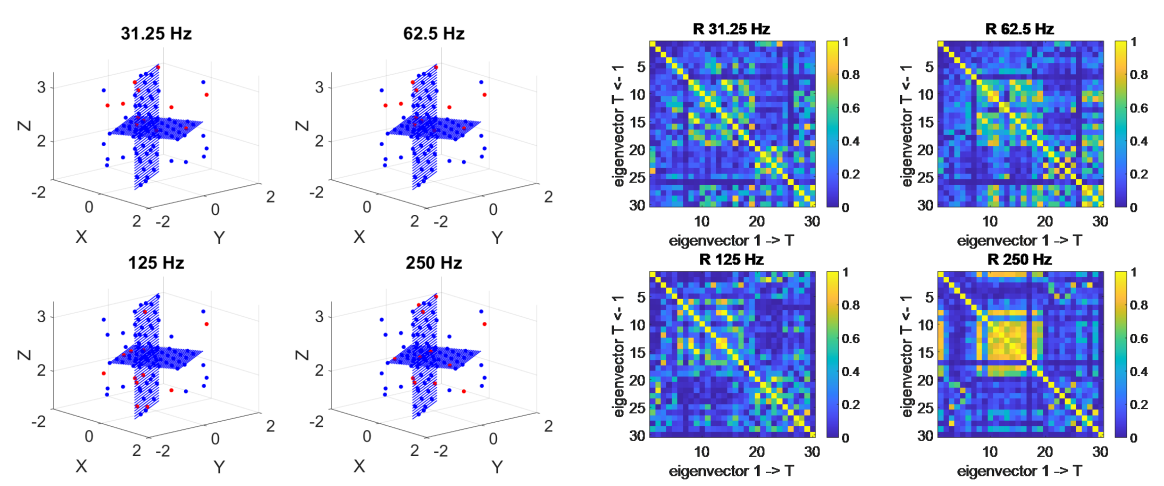

Fig. 15: Optimal spatial distribution of Fig. 16: Modal assurance criterion for microphones in frequency dependent optimal candidate in frequency depenoptimisation.

dent optimisation.
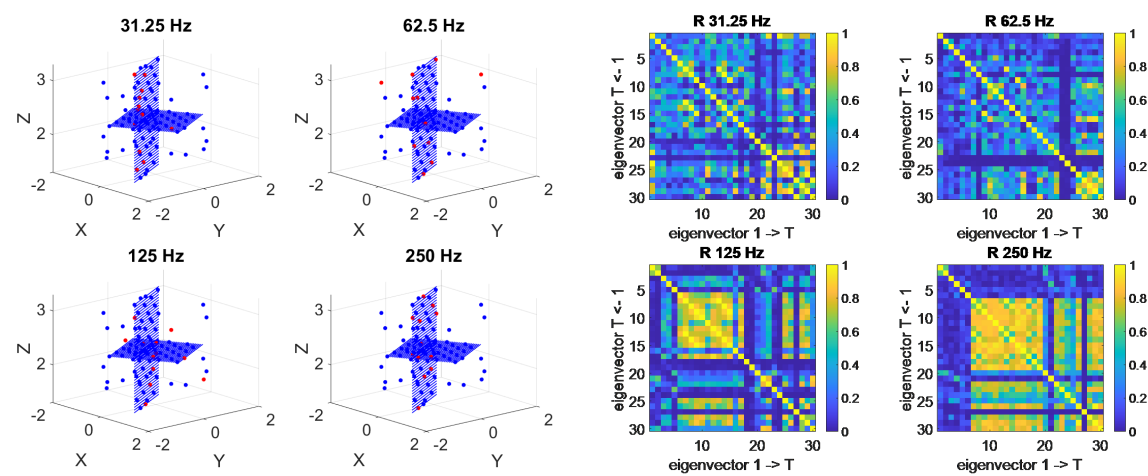

Fig. 17: Worst spatial distribution of Fig. 18: Modal assurance criterion for microphones in frequency dependent worst candidate in frequency depenoptimisation. dent optimisation.

of loudspeakers and subwoofers. Therefore, the control microphones are likely to measure highly coherent responses, which may translate into conditioning issues in the resolution of the inverse problem described in Section 4. For the worst candidates, figure 18 presents important aliasing issues at frequencies 125 $\mathrm{Hz}$ and $250 \mathrm{~Hz}$. This suggests that certain modes may no longer be controlled independently of others.

Figure 19 shows the predicted sound pressure levels for the frequency dependent optimisation. Since the objective of Case study 2 is to analyse its capability to outperform Case 1, only the results for the optimal candidate are presented. On top, the results for the control microphones are shown. $E_{\text {bands }}^{L}$, as well as $E_{\text {std }}^{L}$ show a more uniformly controlled pressure field than in Case study 1. Together with the results in green in figure 14, it is demon- 

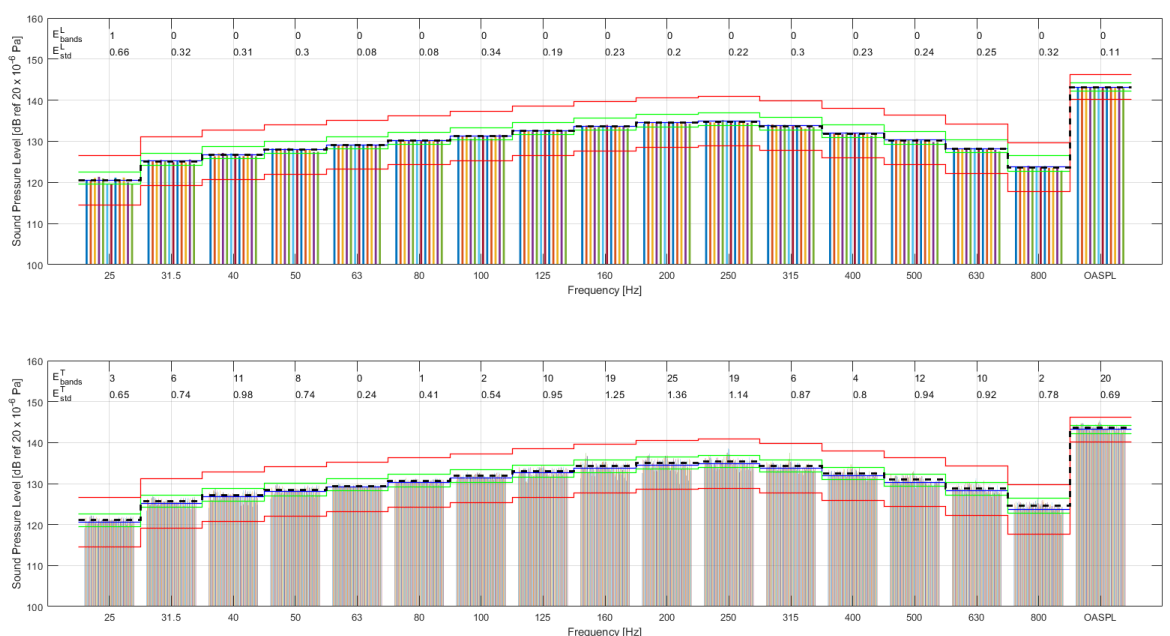

Fig. 19: Sound pressure levels for optimal candidate in frequency dependent optimisation. Top: $L$ control microphones. Bottom: $T$ microphones.

strated that smaller dispersion of sound pressure is realized by Case study 2 . On the bottom, figure 19 also shows enhanced uniformity in spatial distribution of sound pressure level for the $T$ microphones. $E_{b a n d s}^{T}$ decreases for all the $1 / 3$ rd octave bands with respect to Case study 1 , except for the ones with center frequency $50 \mathrm{~Hz}, 200 \mathrm{~Hz}$ and $630 \mathrm{~Hz}$. In the same figure, $E_{\text {std }}^{T}$ slightly decreases with respect to Case study 1 , except for the $1 / 3 \mathrm{rd}$ octave bands with center frequency $80 \mathrm{~Hz}, 100 \mathrm{~Hz}, 200 \mathrm{~Hz}$ and $630 \mathrm{~Hz}$. Although both descriptors show, in general, an enhancement in sound pressure level uniformity, further analysis involving additional optimisation candidates may be needed at 200 $\mathrm{Hz}$ and $630 \mathrm{~Hz}$.

Figure 20 focuses on the control effort required by the different optimisation approaches. This descriptor is displayed as reference in blue for the full-band optimisation in Case study 1. The green dashed curve shows that the optimal candidate from the frequency dependent optimisation requires an equivalent average magnitude for the signals $\boldsymbol{u}$ driving the loudspeakers. Furthermore, the results obtained for the worst candidate in the frequency dependent optimisation is shown in red. The latter requires a much higher voltage, especially at low frequency. Figure 21 shows that such inefficiency presented by the worst candidate in the frequency dependent optimisation is due to conditioning issues. The Condition Number is defined as the ratio $\sigma_{1} / \sigma_{M}$ for the over-determined inverse problem presented in the theoretical section. Then, a candidate with a large spread of singular values will present a large Condition Number. The singular vectors linked to very small singular values, $\sigma_{M}$, could be considered, in practical terms, as belonging to the nullspace of $\boldsymbol{G}$. This implies that certain desired pressure responses can not be 


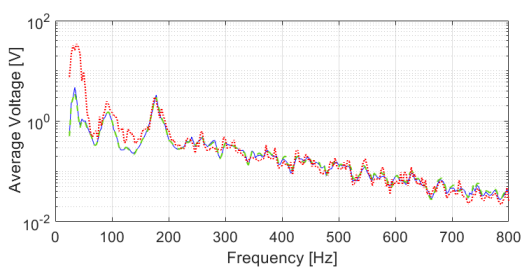

Fig. 20: Control effort for optimisation approaches.

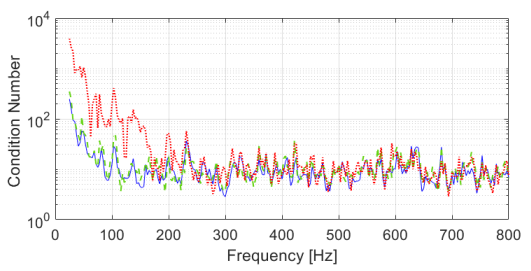

Fig. 21: Condition number for optimisation approaches.

practically reproduced, since they may require a very high control effort as shown by figure 20 .

\section{Conclusions}

The optimisation algorithms in this work are presented as part of a pre-test analysis, which intends to enhance the performance of MIMO control strategies. A frequency dependent optimisation algorithm is proposed for the selection of control microphones. The algorithm is evaluated in the context of functional analysis, spectral decomposition and active acoustic random control, using a numerical model of an electro-acoustic plant. Simulated signals are used to assess the capability of the algorithm to achieve uniform spatial distribution of sound pressure level with reasonable control effort. The results are compared with an approach proposed in a previous work, showing relative improvement for both objectives.

Acknowledgements The authors gratefully acknowledge the European Commission for its support of the Marie Skłodowska-Curie program through the ITN ANTARES project (GA 606817) in collaboration with Siemens Digital Industries Software and KU Leuven. A special thank you also to Thales Alenia Space (France) for kindly providing the structural model of the antenna reflector.

\section{Declarations}

- Funding: The research was partially funded by the Marie Skłodowska-Curie program through the ITN ANTARES project (GA 606817). 
- Conflicts of interest/Competing interests: The authors declare that they have no conflict of interest.

- Availability of data and material: Not applicable.

- Code availability: Not applicable.

\section{References}

1. T. J. Schultz. Diffusion in reverberation rooms. Journal of Sound and Vibration, 16:17-28, 1971.

2. F. Jacobsen and T. Roisin. The coherence of reverberant sound fields. Journal of the Acoustical Society of America, 108:204-210, 2000.

3. I. Veit and H. Sander. The production of a spatially limited'diffuse'sound field in an anechoic room. In Audio Engineering Society Convention 7\%. Audio Engineering Society, 1985.

4. H. Nelisse and N. Jean. Characterization of a diffuse field in a reverberant room. Journal of the Acoustical Society of America, 101:3517-3524, 1997.

5. B. F. Cron and C. H. Sherman. Spatial-correlation functions for various noise models. The Journal of the Acoustical Society of America, 34(11):1732-1736, 1962.

6. S. J. Elliott, M. Cedric, and P. Gardonio. The synthesis of spatially correlated random pressure fields. Journal of the Acoustical Society of America, 117:1186-1201, 2005.

7. M. Alvarez Blanco, E. Matas, R. Hallez, and K. Janssens. Towards a simulation-based digital twin for pre-test analysis on direct field environmental acoustic testing. In 31st Aerospace Testing Seminar, 2018.

8. C. Fabries, B. Brevart, A. Carrella, M. Alvarez Blanco, D. Dal Fitto, and S. Scharfenberg. Experimental validation of direct field acoustic testing. In 14th European Conf. on Spacecraft Structures, Materials and Environmental Testing, 2016.

9. M. Alvarez Blanco, A. Carrella, K. Janssens, and B. Peeters. Pre-test analysis for design of MIMO control strategies on DFAX. In 15th European Conference on Spacecraft Structures, Materials and Environmental Testing, 2018.

10. M. Alvarez Blanco, R.Hallez, K. Janssens, F. Bianciardi, and A. Carrella. Simulation and validation of a mimo direct field acoustic control system for acoustic qualification tests. In 30th Aerospace Testing Seminar, 2017.

11. F. M. Fazi. PhD Dissertation, Sound field reproduction. Southampton University, 2010.

12. D. Colton and R. Kress. Integral equation methods in scattering theory, volume 72. SIAM, 2013.

13. J. R. Higgins. Sampling theory in Fourier and signal analysis: foundations. Oxford University Press on Demand, 1996.

14. F. Marvasti. Nonuniform sampling: theory and practice. Springer Science \& Business Media, 2012.

15. H. Bériot, A. Prinn, and G. Gabard. Efficient implementation of high-order finite elements for helmholtz problems. International Journal for Numerical Methods in Engineering, 106(3):213-240, 2016.

16. D. O. Smallwood. Multiple shaker random control with cross coupling. In Combined Environments: Technology Interrelations, pages 341-347, 1978.

17. B. Peeters and J. Debille. Multiple-input-multiple-output random vibration control: theory and practice. In Proceedings of ISMA 2002: International Conference On Noise And Vibration Engineering, Vols 1-5, pages 507-516. Katholieke Univ Leuven, Dept Werktuigkunde, 2002.

18. S. J. Elliott. Signal processing for active control. Elsevier, 2000.

19. M. A. Poletti. Three-dimensional surround sound systems based on spherical harmonics. Journal of the Audio Engineering Society, 53(11):1004-1025, 2005.

20. W. Heylen, S. Lammens, and P. Sas. Modal Analysis Theory and Testing. Department of Mechanical Engineering, Katholieke Universiteit Leuven, Leuven, Belgium, 2013.

21. M. Alvarez Blanco, K. Janssens, and F. Bianciardi. Projection algorithms for target spectrum matrix definition in mimo direct field acoustic control. In 22nd Int. Congress on Acoustics, 2016. 
22. M. Alvarez Blanco, K. Janssens, and F. Bianciardi. Experimental verification of projection algorithms and optimization routines for acoustic field uniformity enhancement in mimo direct field acoustic control. In ISMA Int. Conf. on Noise and Vibration Engineering, 2016. 